\title{
ANÁLISE DE ESTABILIDADE FENOTÍPICA DE CULTIVARES DE SOJA EM 28 AMBIENTES DO RIO GRANDE DO SUL
}

\author{
PHENOTYPIC STABILITY ANALYSIS OF SOYBEAN CULTIVARS IN \\ 28 ENVIRONMENTS OF THE STATE OF RIO GRANDE DO SUL
}

\author{
Ailo Valmir Saccol* Lindolfo Storck ${ }^{\star \star}$
}

\section{RESUMO}

O presente estudo utilizou os dados de rendimento de 21 cultivares de soja, em 28 ambientes do Rio Grande do Sul, para uma analise de estabilidade pelo modelo bi-segmentado descontínuo (STORCK, 1989), com correçōes devido a erros nas variáveis. As principais conclusões foram: 1) O método mostrou-se adequado para o estudo da estabilidade fenotípica de cultivares de soja, porque houve uma boa variação ambiental e um bom ajustamento dos dados ao modelo; 2) $O$ grupo de cultivares estudado pode ser classificado em: a) Indesejáveis (Hill, IAS-2, Paraná, Prata, Sel. Foscarin, Louisiana, Hardee, Bienville, Industrial, Santa Rosa, Bossier e Viçoja); b) Adaptáveis tanto aos ambientes inferiores como aos superiores, dentro da região estudada (Pérola, Bragg e Jakson); c) Adaptáveis a ambientes superiores (Planalto, Hale-7, IAS-5, IAS-1 e IAS-4); d) Adaptáveis à armbientes médios (Hood).

Palavras-chave: soja, análise da estabilidade, interação cultivares e ambientes

\section{SUMMARY}

Yield data of 21 soybean variety from trials conducted in 28 environments in the Rio Grande do Sul State were used for a stability analyses using the discontinuous bi-segmented model (STORCK, 1989) with corrections due to errors in the variables. The main conclusions were: 1) The method was adequate for phenotype stability studies of soybean varieties since there was a wide environmental variation and a good data adjustment to the model; 2) The group of varieties can be considered as undesirables (Hill, IAS-2, Paraná, Prata, Sel. Foscarin, Louisiana, Hardee, Bienville,
Industrial, Santa Rosa, Bossier and Viçoja); b) Adaptable to both unfavorable and favorable environments, within the region studied (Pérola, Bragg and Jakson); c) Adaptable to favorable environments (Planalto, Hale-7, IAS-5, IAS-1 and (AS-4); d) Adaptable to average environments (Hood).

Key Words: soybean, stability analysis, cultivar-environment interaction

\section{INTRODUÇÃO}

O rendimento de grãos de diferentes cultivares de soja é influenciado pelos fatores ambientais, que podem variar entre locais e/ou entre anos havendo, portanto, interação entre genótipos e ambiente (MUNGOMERY et al, 1974 e VERNETTl et al, 1990). Esta influência, em geral, não é a mesma para as diferentes cultivares dada a natureza genética das mesmas. Tem-se, então, uma interação de cultivares com ambientes, isto é, a forma com que cada cultivar responde à variação ambiental não é a mesma.

O estudo detalhado da interação, via análise da estabilidade, é recomendado para melhor caracterizar as cultivares e permitir determinar quais são as melhores condições ecológicas para cada cultivar. Numa situação desta natureza, segundo MINOR \& BERLATO (1977), seria vantajosa a utilização de cultivares que possuam uma adaptabilidade ampla, garantindo assim, um comportamento satisfatorio em condições diversas. Por outro lado, é também de interesse, em regiōes onde estāo sendo empregados altos niveis de manejo, utilizar cultivares com maiores capacidades de resposta à aplicação de tecnologia.

A identificação destes dois tipos de cultivares, a amplamente adaptada e a adaptada em condiçōes favoráveis, tem sido impedida pela inexistência de métodos

\footnotetext{
* Engenheiro Agrônomo, Professor Titular do Departamento de Fitotecnia (DF), Centro de Ciências Rurais (CCR), Universidade Federal de Santa Maria (UFSM). 97119-900 - Santa Maria - RS. Bolsista do CNPq.

** Engenheiro Agrônomo, Prof. Adjunto, DF, UFSM, Bolsista CNPq.
} 
que possibilitem uma precisa quantificação do comportamento das cultivares frente às variaçōes $e$ interaçōes complexas do ambiente (MINOR \& BERLATO, 1977). Neste sentido, FINLAY \& WILKINSON (1963), desenvolveram um método estatístico para avaliar o comportamento de plantas em diversos ambientes tomando-se, apenas, os dados de rendimento. SANTOS \& VIEIRA (1975) aplicaram este método para o rendimento de grãos da cultura da soja. Este método foi utilizado por EBERHART \& RUSSELL (1966), com algumas modificações, os quais concluiram ser ele adaptável à análise de dados provenientes de ensaios regionais e nacionais de cultivares.

O método de FINLAY \& WILKINSON (1963), modificado por EBERHART \& RUSSELL (1966), foi utilizado por MINOR \& BERLATO (1977), para avaliar o grau de adaptação de algumas cultivares de soja, em 42 ambientes, no Rio Grande do Sul. Como resultado, verificaram que a cultivar Planalto apresentou parâmetros que indicam adaptação à ambientes de baixa produtividade; a Bragg, IAS-1 e IAS-2 apresentaram comportamento estável, enquanto a Hardee e a Bossier mostraram-se sensíveis à variação do ambiente.

Os métodos desenvolvidos mais recentemente, para estudar a interação genótipo e ambiente, em diversas culturas, no entanto, tem-se preocupado em detaIhar a interação, através do desdobramento da variação ambiental, dentro de cada cultivar, em regressões seg. mentadas e desvios (STORCK, 1989). Estes métodos, têm sido, ultimamente, os mais utilizados.

O presente estudo visa: 1) Testar, para a cultura da soja, um modelo de regressão bi-segmentado descontínuo visando avaliar a estabilidade fenotípica de cultivares de soja; 2) Identificar cultivares que apresentam major estabilidade de rendimento frente a variação do ambiente (em ambientes inferiores e/ou superiores) e 3) Identificar cultivares que apresentam alta capacidade de resposta à ambientes superiores.

\section{MATERIAL E MÉTODOS}

O presente trabalho foi realizado a partir dos dados de rendimento de grãos de soja obtidos durante o ano agrícola de 1973/74 em 28 ambientes (Tabela 1). Em cada ambiente foram avaliadas 21 cultivares de soja, as quais estão relacionadas na Tabela 2. Nos ambientes $1,2,4,9,11,13,23,24,26$ e 27 os experimentos foram conduzidos pelo IPAGRO-SA; nos ambientes 5 e 25 os experimentos foram conduzidos pelo Centro de Experimentação e Pesquisa - FECOTRIGO; nos ambientes $3,6,7,8,14$ e 15 os experimentos foram conduzidos peln CNPT - EMBRAPA; nos ambientes 12 e 28 os experimentos foram conduzidos pela UFSM e nos ambientes $16,17,18,19,20,21$ e 22 os experimentos foram
TABELA 1 - Relaçáo das cultivares de soja avaliadas

\begin{tabular}{|c|c|c|c|c|c|}
\hline \multirow{3}{*}{$\begin{array}{l}\text { Cultivar } \\
1 \text { Hill } \\
2 \text { Hood } \\
3 \text { LAS-2 }\end{array}$} & Origem & Instituiçăăo & Ano & \multicolumn{2}{|c|}{$\begin{array}{l}\text { Maturaçăo } \\
\mathbf{A}^{1} \mathbf{B}^{2}\end{array}$} \\
\hline & $\begin{array}{l}\text { D632-15 } \times \text { O49-2525 } \\
\text { Roanok } \times \text { Ogden-CNS }\end{array}$ & Virgínia AES ${ }^{3}$ USASL $^{5}$ & $\begin{array}{l}1959^{*} \\
1958^{*}\end{array}$ & $\begin{array}{c}v \\
v\end{array}$ & $\begin{array}{l}P \\
\text { SP }\end{array}$ \\
\hline & $\begin{array}{l}\text { Sel. em N59-6921 (Hill x } \\
\text { Roanok Ogden) }\end{array}$ & IPEAS - MA - RS & $1973^{*}$ & $\mathrm{v}$ & $\mathrm{P}$ \\
\hline 4 Planalto & $\begin{array}{l}\text { Hood x Kedelee STB } \\
N^{0} 452\end{array}$ & IPAGRO - SARGS & $1972^{*}$ & v & SP \\
\hline $\begin{array}{l}5 \text { Hale-7 } \\
6 \text { IAS-5 } \\
7 \text { Perola } \\
8 \text { Paraná }\end{array}$ & $\begin{array}{l}\text { Hill } \times \text { Hood } \\
\text { Hood } \times \text { Industrial } \\
\text { Hill } \times \text { F1. (Roanok } \times \\
\text { Ogden) }\end{array}$ & $\begin{array}{l}\text { IPEAS - MA - RS } \\
\text { IPAGRO - SARGS } \\
\text { IPEAME - MA - PR }\end{array}$ & $\begin{array}{l}1973^{*} \\
1973^{*} \\
1973^{*}\end{array}$ & $\begin{array}{l}\mathrm{VII} \\
\mathrm{VI} \\
\mathrm{Vl} \\
\mathrm{Vl}\end{array}$ & $\begin{array}{l}\text { M } \\
\text { SP } \\
\text { SP } \\
\text { SP }\end{array}$ \\
\hline $\begin{array}{l}9 \text { Prata } \\
10 \text { Sel. Foscarin }\end{array}$ & $\begin{array}{l}\text { Hood } \times \text { Hill } \\
\text { Sel. }{ }^{4} \text { da cultivar Hale-7 }\end{array}$ & IPAGRO - SARGS & $\frac{1973^{*}}{\cdots---}$ & v & $\mathrm{SP}$ \\
\hline $\begin{array}{l}11 \text { IAS-1 } \\
12 \text { LOUIS }\end{array}$ & $\begin{array}{l}\text { Sel. }{ }^{4} \text { em F59-1509 } \\
\text { (Jakson x D49-2491) }\end{array}$ & IPEAS - MA - RS & $1973^{*}$ & VII & $M$ \\
\hline 13 IAS-4 & $\begin{array}{l}\text { Sel. }{ }^{4} \text { em R.60-390 } \\
\text { (Hood x Jakson }\end{array}$ & IPEAS - MA - RS & $1973^{*}$ & VII & $M$ \\
\hline $\begin{array}{l}14 \text { Bragg } \\
15 \text { Jakson }\end{array}$ & $\begin{array}{l}\text { Jakson } \times \text { D49-2491 } \\
\text { Volstate } \times \text { Volstate } \\
\text { Palmetto }\end{array}$ & $\begin{array}{l}\text { Florida AES }{ }^{3} \text { a } \text { USRSL }^{5} \\
\text { North Carolina AES }{ }^{3} \text { o } \\
\text { USRSL }\end{array}$ & $\begin{array}{l}1963^{*} \\
1953^{*}\end{array}$ & $\begin{array}{l}\mathrm{Vl} \\
\mathrm{Vl}\end{array}$ & $\begin{array}{l}M \\
M\end{array}$ \\
\hline 16 Hardee & $\begin{array}{l}\text { D49-772 x Improved } \\
\text { Pelican }\end{array}$ & Florida AES $^{3}$ e USRSL ${ }^{5}$ & $1962^{*}$ & V:1 & $T$ \\
\hline 17 Bienville & Pelicano no2 $\times$ Ogden & $\begin{array}{l}\text { Louisiana AES }{ }^{3} \text { o } \\
\text { USRSL }^{5}\end{array}$ & $1958^{*}$ & VII & $T$ \\
\hline $\begin{array}{l}18 \text { Industrial } \\
19 \text { Santa Rosa } \\
20 \text { Bossier }\end{array}$ & 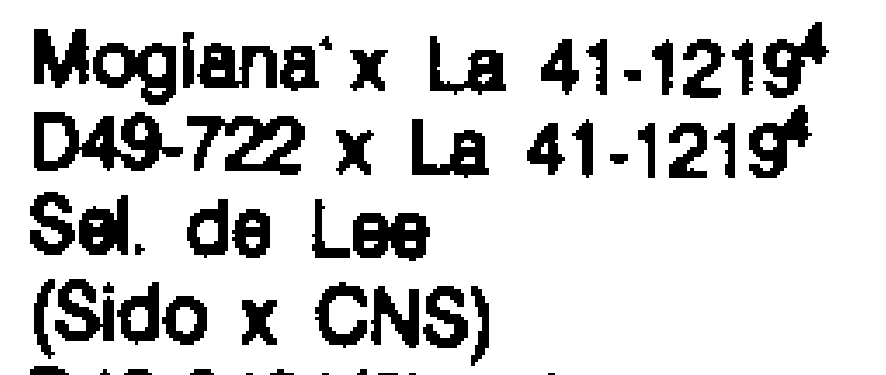 & $\begin{array}{l}\text { IAC - SP } \\
\text { IAC - SP } \\
\text { Louisiana AES }{ }^{3} \text { e } \\
\text { USRSL }\end{array}$ & $1967^{*}$ & $\begin{array}{l}\text { VIII } \\
\text { VII } \\
\text { VIl }\end{array}$ & $\begin{array}{l}\mathrm{T} \\
\mathrm{T} \\
\mathrm{M}\end{array}$ \\
\hline 21 Viçoja & D49-2491 (2) X Pelican & UFV - MG & $1969^{\circ}$ & $\mathrm{VH}$ & $T$ \\
\hline
\end{tabular}

1'Grupo da Classificaçăo Americana; ${ }^{2}$ Grupo da Classificaçăo relativa ao RS ( $P=P r e c o c e, ~ S P=S e$ miprecoce, $M=M b i c$, $T=$ Tardion; gional Soybean Laboratory; Ano de lançamento e Hábito de crescimento determinado; Fonte
SEDIYAMA al (1981).

TABELA 2 - Relaçáo dos ambientes om que as cultivares de soja to ram avaliadas no Rio Grande do Sul, durante o ano agricola de 1973/74.

\begin{tabular}{|c|c|c|c|c|}
\hline No $^{2} \begin{array}{l}\text { Ambiente } \\
\text { Local (*) }\end{array}$ & $\begin{array}{l}\text { Coorder } \\
\text { Lat. }\end{array}$ & $\begin{array}{l}\text { adas Geogi } \\
\text { Lona. }\end{array}$ & & $\begin{array}{l}\text { Nivel de } \\
\text { Fertilidade }\end{array}$ \\
\hline $\begin{array}{l}1 \text { Santo Augusto (2) } \\
2 \text { Santa Rosa (1) } \\
3 \text { Erexim (2) } \\
4 \text { Júlio de Castilhos(1) } \\
5 \text { Cruz Alta (1) } \\
6 \text { Passo Fundo (1) } \\
7 \text { Tapera (3) } \\
8 \text { Passo Fundo (1) } \\
9 \text { Viamáo (1) } \\
10 \text { Gravata (3) } \\
11 \text { Cachoeira do Sul(1) } \\
12 \text { Santa Maria (1) } \\
13 \text { Veranopolis (2) } \\
14 \text { Encantado (3) } \\
15 \text { Vacaria (1) } \\
16 \text { Camaqua (3) } \\
17 \text { Pelotas (Sede)(1) } \\
18 \text { Pelotas (Palma)(1) } \\
19 \text { Arroio Grande (3) } \\
20 \text { Pedro Osório (3) } \\
21 \text { Pelotas (Medonho)(1) } \\
22 \text { Osório (2) } \\
23 \text { Bagé (1) } \\
24 \text { Sáo Borja (1) } \\
25 \text { Cruz Alta (1) } \\
26 \text { Santa Rosa (1) } \\
27 \text { Veranopolis (2) } \\
28 \text { Santa Maria (1) }\end{array}$ & $\begin{array}{l}27^{\circ} 54^{\prime} 16^{\prime \prime} \\
27^{\circ} 51^{\prime} 50^{\prime \prime} \\
27^{\circ} 37^{\prime} 45^{\prime \prime} \\
29^{\circ} 13^{\prime} 26^{\prime \prime} \\
28^{\circ} 38^{\prime} 21^{\prime \prime} \\
28^{\circ} 15^{\prime} 39^{\prime \prime} \\
28^{\circ} 36^{\prime} 14^{\prime \prime} \\
28^{\circ} 15^{\prime} 39^{\prime \prime} \\
30^{\circ} 05^{\prime} 00^{\prime \prime} \\
29^{\circ} 54^{\prime} 20^{\prime \prime} \\
30^{\circ} 02^{\prime} 45^{\prime \prime} \\
29^{\circ} 41^{\prime} 25^{\prime \prime} \\
28^{\circ} 36^{\prime} 14^{\prime \prime} \\
29^{\circ} 10^{\prime} 11^{\prime \prime} \\
28^{\circ} 33^{\prime} 00^{\prime \prime} \\
30^{\circ} 53^{\prime} 51^{\prime \prime} \\
31^{\circ} 45^{\prime} 00^{\prime \prime} \\
31^{\circ} 45^{\prime} 00^{\prime \prime} \\
32^{\circ} 09^{\prime} 08^{\prime \prime} \\
31^{\circ} 46^{\prime} 25^{\prime \prime} \\
31^{\circ} 45^{\prime} 00^{\prime \prime} \\
29^{\circ} 40^{\prime} 49^{\prime \prime} \\
31^{\circ} 20^{\prime} 14^{\prime \prime} \\
28^{\circ} 39^{\prime} 44^{\prime \prime} \\
28^{\circ} 38^{\prime} 21^{\prime \prime} \\
27^{\circ} 51^{\prime} 50^{\prime \prime} \\
28^{\circ} 56^{\prime} 14^{\prime \prime} \\
29^{\circ} 41^{\prime} 25^{\prime \prime}\end{array}$ & $\begin{array}{l}53^{\circ} 45^{\prime} 14^{\prime \prime} \\
54^{\circ} 25^{\prime} 59^{\prime \prime} \\
52^{\circ} 16^{\prime} 33^{\prime \prime} \\
53^{\circ} 40^{\prime} 45^{\prime \prime} \\
53^{\circ} 36^{\prime} 34^{\prime \prime} \\
52^{\circ} 24^{\prime} 33^{\prime \prime} \\
52^{\circ} 47^{\prime} 44^{\prime \prime} \\
52^{\circ} 24^{\prime} 33^{\prime \prime} \\
50^{\circ} 47^{\prime} 00^{\prime \prime} \\
51^{\circ} 06^{\prime} 10^{\prime \prime} \\
52^{\circ} 53^{\prime} 39^{\prime \prime} \\
53^{\circ} 48^{\prime} 42^{\prime \prime} \\
51^{\circ} 33^{\prime} 11^{\prime \prime} \\
51^{\circ} 47^{\prime} 44^{\prime \prime} \\
50^{\circ} 42^{\prime} 21^{\prime \prime} \\
51^{\circ} 43^{\prime} 13^{\prime \prime} \\
52^{\circ} 21^{\prime} 00^{\prime \prime} \\
52^{\circ} 21^{\prime} 00^{\prime \prime} \\
53^{\circ} 04^{\prime} 05^{\prime \prime} \\
52^{\circ} 47^{\prime} 44^{\prime \prime} \\
52^{\circ} 21^{\prime} 00^{\prime \prime} \\
50^{\circ} 13^{\prime} 36^{\prime \prime} \\
54^{\circ} 05^{\prime} 59^{\prime \prime} \\
56^{\circ} 00^{\prime} 15^{\prime \prime} \\
53^{\circ} 40^{\prime} 45^{\prime \prime} \\
54^{\circ} 25^{\prime} 59^{\prime \prime} \\
51^{\circ} 33^{\prime} 11^{\prime \prime} \\
53^{\circ} 48^{\prime} 42^{\prime \prime}\end{array}$ & $\begin{array}{r}380 \\
360 \\
760 \\
514 \\
473 \\
678 \\
450 \\
678 \\
52 \\
10 \\
68 \\
86 \\
705 \\
400 \\
955 \\
5 \\
7 \\
7 \\
10 \\
10 \\
7 \\
32 \\
214 \\
96 \\
473 \\
350 \\
705 \\
96\end{array}$ & $\begin{array}{l}\text { Corrigida } \\
\text { Corrigida } \\
\text { Corrigida } \\
\text { Corrigida } \\
\text { Corrigida } \\
\text { Corrigida } \\
\text { Corrigida } \\
\text { Natural } \\
\text { Corrigida } \\
\text { Corrigida } \\
\text { Corrigida } \\
\text { Corrigida } \\
\text { Corrigida } \\
\text { Corrigida } \\
\text { Corrigida } \\
\text { Corrigida } \\
\text { Corrigida } \\
\text { Corrigida } \\
\text { Corrigida } \\
\text { Corrigida } \\
\text { Corrigida } \\
\text { Natura } \\
\text { Corrigida } \\
\text { Corrigida } \\
\text { Natura } \\
\text { Natura } \\
\text { Natural } \\
\text { Natural }\end{array}$ \\
\hline
\end{tabular}


conduzidos pelo CNPTBA - EMBRAPA.

As 21 cultivares, em cada experimento, foram avaliadas segundo 0 delineamento experimental Látice Parcialmente Balanceado, com quatro repetiçōes, em unidades experimentais de $4,80 \mathrm{~m}$.

$O$ rendimento de grãos $(\mathrm{kg} / \mathrm{ha})$ das 21 cultivares em 28 ambientes foi analisado conjuntamente e a variação da interação Cultivares $\times$ Ambientes mais a variação de Ambientes foi desdobrada em variação do ambiente dentro de cada cultivar. A variação ambiental, para cada cultivar, foi desdobrada para a análise da estabilidade, em regressão e desvios segundo o modelo de regressão bi-segmentado descontínuo (STORCK, 1989) caracterizado por

$$
\begin{gathered}
\bar{Y}_{i j}=\beta_{0 j}+\beta_{1} \tau_{j}+\beta_{2 i} \tau{ }_{j} Z_{j}+\beta_{3} Z_{j}+\delta_{i j}+\varepsilon_{i j} \\
\text { onde: } \hat{\tau}_{j}=\tau_{j}+v_{j} \\
\hat{\tau}_{j} Z_{j}=\tau_{j} Z_{j}+v_{j} Z_{j} \\
z_{j}=\mid \begin{array}{l}
1, \text { se } \hat{\tau} j>0 \\
0, \text { se } \hat{\tau} \leq 0,
\end{array}
\end{gathered}
$$

$Y_{i j}$ é a média da i-ésima cultivar no j-ésimo ambiente; $\beta_{0 j}$ é o valor da função no ponto $\boldsymbol{\tau}_{j}=0$ do primeiro segmento; $\beta_{1}$ é a inclinação do primeiro segmento de reta; $\beta_{2}$ é a diferenca de inclinação entre os dois segmentos de reta, tal que $\beta_{1} i+\beta_{2}$ é a inclinação do segundo segmento de reta; $\beta_{3} i$ mede a descontinuidade entre os dois segmentos de reta; $\delta_{i j}$ e o desvio da observação ao modelo, tal que: $E\left(\delta_{i j}\right)=0$ e $E\left(\delta_{i j}^{2}\right)=\sigma_{i \delta i}^{2}$ ; $\varepsilon_{i j}$ é o erro associado ao estimador $\bar{Y}_{i j}$, independente de $\delta_{i j}$, com esperança zero e variância $\sigma_{\varepsilon}^{2}$ estimada a partir do quadro da análise de variância conjunta por $\hat{\boldsymbol{\sigma}}_{\varepsilon}^{2}=\mathrm{QMR} / \mathrm{K}_{i} \boldsymbol{\tau}_{\mathrm{j}}$ é o índice ambientâl j (não observável), de efeito aleatório, estimado por $\hat{\tau}_{j}=\bar{Y}_{\cdot j}$. $-\bar{Y}_{. . . e}$ associado ao erro de estimativa $u_{j}$ tal que $\operatorname{Var}$ $\left(v_{j}\right)=\sigma_{u}^{2}$ estimada por $\hat{\sigma}^{2}{ }_{v}=(\mathrm{J}-1)(\mathrm{QMR}) / \mathrm{JK}$ e $\operatorname{Cov}\left(\varepsilon_{i j} ; v_{j}\right)=\sigma_{\varepsilon u}=\sigma_{u}^{2}$. Como $\sigma_{\varepsilon}^{2}$ e $\sigma_{u}^{2}$ são estimáveis a partir da análise conjunta, as estimativas de todos os parâmetros, para cada cultivar, pode ser obtido pelo método dos momentos. Estas estimacões, bem como os testes de hipóteses, foram obtidos conforme descrição em STORCK (1989).

\section{RESULTADOS E DISCUSSÃO}

Os resultados da análise da variância (Tabela 3) mostram que a variância ambiental é significativa, isto é,
TABELA 3 - Quadro da análise da variancia com desdobramento da interaçăo re ferente ao grupo de experimentos com soja $(\mathrm{kg} / \mathrm{ha})$.

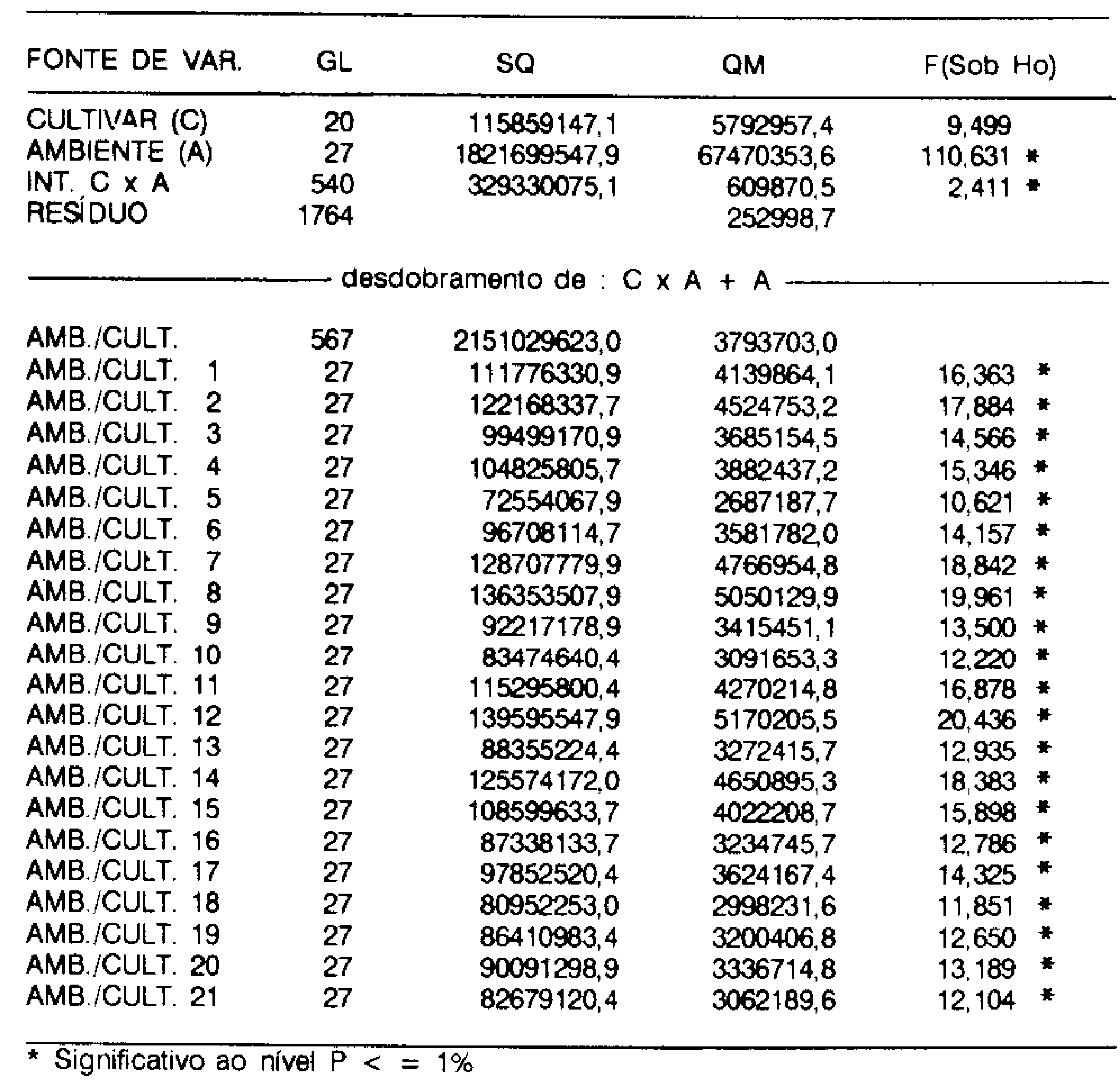

as cultivares são sensiveis a variação ambiental quanto à produtividade de grãos. Além disso, dado que a interação foi significativa, pode-se dizer que a sensibilidade, quanto à variação ambiental, não foi a mesma para as diferentes cultivares. Interaçōes significativas, para a cultura da soja, tambem, foram observadas por MUNGOMERY et al (1974) e VERNETTI et al (1990). Como a variação ambiental foi significativa para todas as cultivares, este conjunto de experimentos é apropriado para uma análise de estabilidade, podendo-se, portanto, desdobrar a fonte de variação ambiente dentro de cada cultivar em regressäo e desvios, tomando-se, para isto, a variação ambiental como variavel independente.

A caracterização dos valores ambientais, tais como o Quadrado Médio do Resíduo (QMR), Média, Coeficiente de Variação (CV) e Variáveis Independentes (índice ambiental $\hat{\tau}$ e suas funções $Z$ e $\bar{Z} \hat{\imath}$ ) estão apresentadas na Tabela 4. Nesta, observa-se que 0 índice ambiental varia entre $-1782,08$ (Ambiente 22) a 1377,83 (Ambiente 18) $\mathrm{kg} / \mathrm{ha}$, o que mostra uma amplitude de variação de $3159,91 \mathrm{~kg} / \mathrm{ha}$, com índices $\boldsymbol{\tau}$, adequados $\mathrm{e}$ relativamente, equidistantes. Estudo neste sentido, desenvolvido por VERNETTI et al (1990) em 35 ambientes, mostrou uma amplitude de variação, para os índices ambientais, de $2417,8 \mathrm{~kg} / \mathrm{ha}$ com extremos entre $-982,9 \mathrm{e}$ $1434,9 \mathrm{~kg} / \mathrm{ha}$. Assim, a amplitude obtida neste trabalho é adequada para o estudo do comportamento de cada cultivar em função da variação ambiental. Os coeficientes de variaçāo, oscilando entre $9,00 \%$ a $27,00 \%$, e com média de $17,45 \%$, variam proporcionalmente menos do que os QMR, os quais são significativamente heterogêneos pelo teste de Bartlett. Os resulta- 
TABELA 4 - Quadrado médio de resíduo (QMR), média, coeficiente de variaçāo (CV) e variáveis independentes ( $\hat{\tau}, \hat{\tau} Z$ e $z$ ) para os ambientes do grupo de experimentos com soja $(\mathrm{kg} / \mathrm{ha})$.

\begin{tabular}{lrrrrrr}
\hline Amb. & QMR & Media & \multicolumn{1}{c}{ CV } & \multicolumn{1}{c}{$\hat{\boldsymbol{\tau}}$} & \multicolumn{1}{c}{$\hat{\boldsymbol{\tau}} \mathrm{z}$} & $\mathrm{z}$ \\
\hline & & & & & & \\
1 & 285712 & 3818,0 & 14,00 & 990,30 & 990,30 & 1 \\
2 & 306528 & 2214,6 & 25,00 & $-613,12$ & 0,00 & 0 \\
3 & 162091 & 3414,8 & 11,79 & 587,07 & 587,07 & 1 \\
4 & 83255 & 3206,0 & 9,00 & 378,35 & 378,35 & 1 \\
5 & 203752 & 2201,9 & 20,50 & $-625,84$ & 0,00 & 0 \\
6 & 319329 & 3424,8 & 16,50 & 597,11 & 597,11 & 1 \\
7 & 220739 & 4121,3 & 11,40 & 1293,64 & 1293,64 & 1 \\
8 & 120485 & 1448,1 & 23,97 & $-1379,60$ & 0,00 & 0 \\
9 & 158684 & 2489,7 & 16,00 & $-337,98$ & 0,00 & 0 \\
10 & 75405 & 2112,3 & 13,00 & $-715,41$ & 0,00 & 0 \\
11 & 119149 & 3451,8 & 10,00 & 624,11 & 624,11 & 1 \\
12 & 570753 & 2905,7 & 26,00 & 78,02 & 78,02 & 1 \\
13 & 101511 & 1991,3 & 16,00 & $-836,41$ & 0,00 & 0 \\
14 & 192778 & 3276,6 & 13,40 & 448,88 & 448,88 & 1 \\
15 & 97583 & 2947,0 & 10,60 & 119,26 & 119,26 & 1 \\
16 & 561530 & 3437,4 & 21,80 & 609,69 & 609,69 & 1 \\
17 & 486598 & 4009,0 & 17,40 & 1181,35 & 1181,35 & 1 \\
18 & 447126 & 4205,5 & 15,90 & 1377,83 & 1377,83 & 1 \\
19 & 219275 & 3274,6 & 14,30 & 446,92 & 446,92 & 1 \\
20 & 180809 & 2853,8 & 14,90 & 26,11 & 26,11 & 1 \\
21 & 488838 & 4009,0 & 17,44 & 1181,35 & 1181,35 & 1 \\
22 & 62973 & 1045,6 & 24,00 & $-1782,08$ & 0,00 & 0 \\
23 & 211968 & 2877,5 & 16,00 & 49,78 & 49,78 & 1 \\
24 & 851820 & 3418,3 & 27,00 & 590,64 & 590,64 & 1 \\
25 & 181100 & 1961,1 & 21,70 & $-866,60$ & 0,00 & 0 \\
26 & 104383 & 139,6 & 23,20 & $-1435,12$ & 0,00 & 0 \\
27 & 53908 & 178,6 & 13,00 & $-1041,65$ & 0,00 & 0 \\
28 & 215883 & 1881,1 & 24,70 & $-946,60$ & 0,00 & 0 \\
& & & & & & \\
Media & 252999 & 2827,7 & 17,45 & 0,00 & 377,87 & 0,61 \\
& & & & & &
\end{tabular}

dos mostram ainda que existe uma correlação positiva $(r=0,534)$ entre as médias e os QMR, de tal forma que, ao desejar-se uma boa amplitude de variação nas médias tem-se que tolerar uma certa heterogenidade nos QMR ou nos coeficientes de variação.

As estimativas dos parâmetros de estabilidade, com suas médias e testes de hipóteses, para as $21 \mathrm{cul}$ tivares, estão representadas nas Tabelas 5 e 6 . Na Tabela 6, o termo "Média Inf" é definido como sendo a média da cultivar calculada sobre os 11 ambientes com índice ambiental negativo ( $\boldsymbol{\tau}_{\mathbf{j}} \leq \mathbf{0}$, Tabela 4) ou seja para ambientes de tecnologia inferior e, o termo "Med Sup" como sendo a média da cultivar calculada sobre os 17 ambientes com índice ambiental positivo $\left(\tau_{j}>0\right.$, Tabela 4) ou seja, de tecnologia superior. Os ambientes inferiores são, em geral, os caracterizados pela fertilidade não corrigida e/ou deficiência hídrica.

Os coeficientes de determinação (Tabela 5) são relativamente altos (média de $86,275 \%$ ) e há uma expressiva variação na mudança dos ângulos $\left(\beta_{2}\right)$ do segundo segmento em relação ao ângulo do primeiro segmento $\left(B_{1}\right)$. Estas evidências dão prova da adequação do modelo bi-segmentado, se comparado com os modelos de regressão linear de EBERHART \& RUSSELL (1966), na análise do comportamento das cultivares frente à variação ambiental, na cultura da soja:
TABELA 5 - Estimativas dos parâmetros ( $B O, B 1, B 2$ e $B 3)$ do modelo bi-segmentado descontínuo e do coeficiente de determinaçăo (R2) para as cultivares de soja (kg/ha).

\begin{tabular}{|c|c|c|c|c|c|}
\hline Cult. & $B \varnothing$ & B1 & B2 & B3 & $\mathrm{R} 2(\%)$ \\
\hline 1 & 1866,38 & 0,5214 & 0,5978 & $328,60^{*}$ & 71,513 \\
\hline 2 & 2550,49 & 0,7960 & 0,0529 & 589,43 & 88,758 \\
\hline 3 & 2756,56 & 1,0115 & $-0,1826$ & 145,33 & 87,765 \\
\hline 4 & 3132,01 & 1,1393 & $-0,2909$ & 79,18 & 91,388 \\
\hline 5 & 2994,01 & 1,0649 & $-0,1911$ & $-229,50$ & 90,395 \\
\hline 6 & 3014,64 & 1,1369 & $-0,2484$ & $-70,29$ & 88,434 \\
\hline 7 & 2942,71 & 0,9700 & 0,1513 & 248,02 & 90,722 \\
\hline 8 & 2557,67 & 0,9521 & 0,2266 & 164,25 & 81,933 \\
\hline 9 & 2685,19 & 0,8954 & 0,0680 & 86,19 & 88,225 \\
\hline 10 & 3250,74 & $1,4333^{\circ}$ & $-0,4997$ & $627,09^{*}$ & 87,377 \\
\hline 11 & 3542,84 & 1,4578 & $-0,2386$ & $-550,93$ & 89,192 \\
\hline 12 & 2889,92 & 0,8987 & 0,5059 & 54,96 & 83,307 \\
\hline 13 & 3115,36 & 1.0938 & $-0,4065^{*}$ & 116,24 & 93.477 \\
\hline 14 & 2809,17 & 0,8306 & 0,2248 & 422,30 & 90,101 \\
\hline 15 & 2858,94 & 0,9969 & 0,1302 & 17,30 & 90,405 \\
\hline 16 & 2558,07 & 0,9528 & 0,1276 & $-130,83$ & 89,202 \\
\hline 17 & 2733,93 & 0,8619 & 0,0443 & 146,03 & 80,688 \\
\hline 18 & 2924,76 & 1.0881 & 0.1311 & $-537,82^{*}$ & 86,363 \\
\hline .19 & 2717,78 & 0,9061 & 0,0990 & $-164,89$ & 76,349 \\
\hline 20 & 2879,93 & 1,1213 & $-0,1569$ & $-217,17$ & 86,262 \\
\hline 21 & 2600,50 & 0,8712 & $-0,1447$ & 130,71 & 79,925 \\
\hline Média & 2827,70 & 1,0000 & $-0,0000$ & $-0,00$ & 86,275 \\
\hline
\end{tabular}

TABELA 6 - Média geral, médias nos ambientes inferiores e média nos ambientes superiores e variância dos desvios do modelo (VD), para as cultivares de soja ( $\mathrm{kg} / \mathrm{ha})$.

\begin{tabular}{|c|c|c|c|c|}
\hline Cult. & Média Geral & Média Inf & Média Sup & VD \\
\hline 1 & 2291,79 & 1364,91 & 2891,53 & 271048,952 \\
\hline 2 & 2928,36 & 1784,82 & 3668,29 & 82635,578 \\
\hline 3 & 2775,79 & 1783,64 & 3417,76 & 66408,045 \\
\hline 4 & 3070,14 & 2036,18 & 3739,18 & 33628,738 \\
\hline 5 & 2782,46 & 1969,73 & 3308,35 & 12213,253 \\
\hline 6 & $\mathbf{2 8 7 8 , 1 1}$ & 1921,09 & 3497,35 & 56121,330 \\
\hline 7 & 3150,46 & 2009,73 & 3888,59 & 64017,786 \\
\hline 8 & 2743,04 & 1641,91 & 3455,53 & 196203,897 \\
\hline 9 & 2763,21 & 1823,91 & 3371,00 & 52746,053 \\
\hline 10 & 2681,18 & 1872,09 & 3204,71 & 49189,741 \\
\hline 11 & 3118,18 & 2140,64 & 3750,71 & 69131,033 \\
\hline 12 & 3114,46 & 2025,45 & 3819,12 & 182097,575 \\
\hline 13 & 3032,32 & 2063,27 & 3659,35 & 000000,000 \\
\hline 14 & 3150,50 & 2010,27 & 3888,29 & 69096,292 \\
\hline 15 & 2918,64 & 1900,09 & 3577,71 & 48171,988 \\
\hline 16 & 2526,86 & 1641,64 & 3099,65 & 37877,806 \\
\hline 17 & 2839,32 & 1904,91 & 3443,94 & 136468,778 \\
\hline 18 & 2647,75 & 1878,18 & 3145,71 & 54551,496 \\
\hline 19 & 2655,07 & 1846,27 & 3178,41 & 152531,152 \\
\hline 20 & 2688,79 & 1801,36 & 3263,00 & 68558,788 \\
\hline 21 & 2625,18 & 1762,55 & 3183,35 & 112398,350 \\
\hline Média & 2827,70 & 1865,84 & 3450,07 & 86433,173 \\
\hline
\end{tabular}

Os resultados apresentados na Tabela 6, revelam que as cultivares Hill, IAS-2, Paraná, Prata, Sel. Foscarin, Louisiana, Hardee, Bienville, Industrial, Santa Rosa, Bossier e Viçoja, independente do modelo de resposta 
ao ambiente, definem um comportamento indesejável, para o cultivo econômico, devido apresentarem média baixa tanto no ambiente inferior como no ambiente superior e/ou por possuirem alta variância dos desvios. Em função dos ângulos do primeiro e do segundo segmentos (Tabela 5), as cultivares Pérola, Bragg e Jakson definem um comportamento sujeito a perdas menos intensas $\left(\beta_{1}<1\right)$ de produtividade em ambientes inferiores e de uma melhor resposta $\left(\beta_{2}>0\right)$ nos ambientes superiores, sendo, por isto, indicadas para qualquer ambiente (local) da região onde as cultivares foram avaliadas; e, as cultivares Planalto, Hale-7, IAS-5, IAS-1 e IAS-4 por sua vez, definem um comportamento sujeito a perdas mais intensas $\left(\beta_{1}>1\right)$ em ambientes inferiores e de uma menor resposta $\left(B_{2}<0\right)$, mas ainda positiva, nos ambientes superiores, sendo assim indicadas apenas para os ambientes seguramente superiores; enquanto a cultivar Hood define um tipo de comportamento sujeito a variação de produtividades menor do que a média e semelhante nos ambientes inferiores e superiores $e$, por isto, pode ser recomendada para o cultivo em ambientes médios.

A caracterização do comportamento de cada cultivar analisada, frente a variação ambiental, deve ser interpretada somente dentro do grupo de cultivares analisadas, porque o índice ambiental foi determinado em função destas cultivares.

\section{CONCLUSÕES}

1 - O método mostrou-se adequado para o estudo da estabilidade fenotípica de cultivares de soja;

2 - O grupo de cultivares estudado pode ser classificado em: a) Indesejáveis (Hill, IAS-2, Paraná, Prata, Sel. Foscarin, Louisiana, Hardee, Bienville, Industrial, Santa Rosa, Bossier e Viçoja); b) Adaptáveis tanto aos ambientes inferiores como os superiores, dentro da regiāo estudada (Pérola, Bragg e Jakson); c) Adaptáveis à ambientes superiores (Planalto, Hale-7, IAS-5, IAS-1 e IAS-4); d) Adaptáveis em ambientes médios (Hood).

\section{REFERÊNCIAS BIBLOGRÁFICAS}

EBERHART, S.A., RUSSELL, W.A. Stability parameters for comparing varieties. Crop Science v. 6, p. 36-40, 1966.

FINLAY, K.W., WILKINSON, G.N. The analysis of adaptation in a plant-breeding programme. Australian Journal of Agricultural Research v. 14, p. 742-754, 1963.

IPAGRO - INSTITUTO DE PESQUISAS AGRONÔMICAS. Observaçós meteorológicas no Estado do Rio Grande do Sul. Porto Alegre: Secretaria da Agricultura, 1979. 271 p. Boletim Técnico 3.

MACHADO, F. P. Contribuiçăo ao Estudo do Clima do Rio Grande do Sul. Rio de Janeiro: IBGE, 1950.

MINOR, H. C., BERLATO, M.A. Comportamento de seis cultivares de soja em 42 ambientes do Rio Grande do Sul. Agronomia Sulriograndense v. 13, n. 1, p. 83-92, 1977.

MUNGOMERY, V.E., SHORTER, R., BYTH, D.E. Genotype $X$ Environment Interractions and Environmental Adaptation. I Pattern Analysis Application to Soya Bean Populations. Austr J Agric Res, v. 25, p. 59-72, 1974.

SANTOS, O.S. dos, VIEIRA, C. Análise de adaptação de dez variedades de soja a diferentes ambientes do Estado do Rio Grande do Sul. Revista Ceres, v. 22, n. 124, p. $449-453,1975$.

SEDIYAMA, T., DESTRO, D., SEDIYAMA, C.S. et al. Caracterizaçåo de cultivares de soja. Viçosa: Universidade Federal de Viçosa, 1981. 81 p.

STORCK, L. Modelo de regressão bi-segmentado descontrnuo com erros de medida aplicado na análise de estabilidade de cultivares Piracicaba, 1989. $217 \mathrm{p}$. Tese (Doutorado em Agronomia) - Curso de Pós-Graduação em Estatística e Experimentação Agronômica, Escola Superior de Agricultura "Luiz de Queiróz" / USP, 1989.

VERNETTI, F. de J., GASTAL, M. F. da C., ZONTA, E. $P$. Estabilidade fenotípica de cultivares de soja no Sudeste do Rio Grande do Sul. Pesquisa Agropecuária Brasileira, v. 25, n. 11, p. 1593-1602, 1990. 